\title{
Robust Surface Registration Using a Gaussian-Weighted Distance Map in PET-CT Brain Images
}

\author{
Ho Lee ${ }^{1}$ and Helen Hong ${ }^{2, *}$ \\ ${ }^{1}$ School of Electrical Engineering and Computer Science, Seoul National University \\ holee@cglab.snu.ac.kr \\ ${ }^{2}$ School of Electrical Engineering and Computer Science, BK21: Information Technology, \\ Seoul National University, San 56-1 Shinlim 9-dong Kwanak-gu, Seoul 151-742, Korea \\ hlhong@cse.snu.ac.kr
}

\begin{abstract}
In this paper, we propose a robust surface registration using a Gaussian-weighted distance map for PET-CT brain fusion. Our method is composed of three steps. First, we segment the head using the inverse region growing and remove the non-head regions segmented with the head using the region growing-based labeling in PET and CT images, respectively. The feature points of the head are then extracted using sharpening filter. Second, a Gaussian-weighted distance map is generated from the feature points of CT images to lead our similarity measure to robust convergence on the optimal location. Third, weighted cross-correlation measures the similarities between the feature points extracted from PET images and the Gaussian-weighted distance map of CT images. In our experiments, we use software phantom and clinical datasets for evaluating our method with the aspect of visual inspection, accuracy, robustness, and computation time. Experimental results show that our method is more accurate and robust than the conventional ones.
\end{abstract}

\section{Introduction}

Computed tomography (CT) is a well-established means of diagnosing metastasis of oncology patients and evaluating disease progression and regression during treatment. However, CT has lower sensitivity and specificity than positron emission tomography (PET) in identifying tumors of initial staging or defining their biological behavior and response to therapy, while PET has a limitation in achieving precise lesion size and shape due to the few anatomical structures. Currently, whole body PET-CT fusion using hardware is introduced so as to provide a rough alignment of whole body rapidly. However, it is still critical to develop a registration technique for aligning two different modalities exactly and robustly since images obtained from the PET-CT scanner are acquired with different scan time.

Surface- and voxel-based approaches have been suggested for alignment of functional and anatomical images [1]. In surface-based approach, it requires the delineation of corresponding surfaces in each image. Hongjian et al. [2] used the chamfer distance matching for PET-MR brain fusion. Each rigid surface segmented

* Corresponding author.

M. Lazo and A. Sanfeliu (Eds.): CIARP 2005, LNCS 3773, pp. 794-803 2005.

(C) Springer-Verlag Berlin Heidelberg 2005 
from PET and MR brain images is aligned by repeatedly minimizing values of each distance map. Maintz et al. [3] proposed a feature-based cross-correlation to search for the optimal location where the number of corresponding points between feature points extracted from both images is a maximum. However, the accuracy of these surface-based approaches is largely affected by the result of surface extraction. In voxel-based approach, it measures the similarity of all geometrically corresponding voxel pairs in overlapping area. Especially, mutual information-based registration [4] shows the accurate results in comparison with other voxel-based approaches and surface-based approach. However, mutual information-based registration requires enormous processing time in comparison with surface-based approaches even though multi-resolution technique or other improvements are used.

Current approaches still need more progress in computational efficiency and accuracy for registration between functional and anatomical images. In this paper, we propose a surface-based registration using Gaussian-weighted distance map (GWDM) to robustly find optimal location even in bad conditions such as blurry and noisy images. Our method is applied to PET and CT brain images, which divided into three steps such as head segmentation and non-head elimination, Gaussian-weighted distance map generation, similarity measure and optimization. In our experiments, we use software phantom and clinical datasets for evaluating our method with the aspects of visual inspection, accuracy, robustness, and computation time.

The organization of the paper is as follows. In Section 2, we discuss how to extract feature points efficiently. Then we propose a robust surface registration using Gaussian-weighted distance map in PET and CT brain images. In Section 3, experimental results show how our method aligns exactly and robustly using software phantom and clinical datasets. This paper is concluded with a brief discussion of the results in Section 4.

\section{Surface Registration Using GWDM}

Fig. 1 shows the pipeline of our method for the registration of PET and CT brain images. Since CT images have more anatomical information than PET images, CT images are fixed as reference volume and PET images are defined as floating volume. Since rigid transformation is enough to align the head base, we use three translations and three rotations about the $x-, y-, z-$ axis.

\subsection{Head Segmentation Using 3D Inverse Region Growing}

Since the head segmentation using threshold-based method can produce holes within the head, these holes should be filled by morphological operations such as dilation and erosion. However, we decide the number of iterations of morphological operation in proportion to the size of holes as well as the computation time is increased by the number of iterations. In addition, numerous iterations can produce distortions of edge. Thus we propose a 3D inverse region growing (IRG) for the automatic head segmentation without additional processing such as hole filling in PET and CT brain images. 


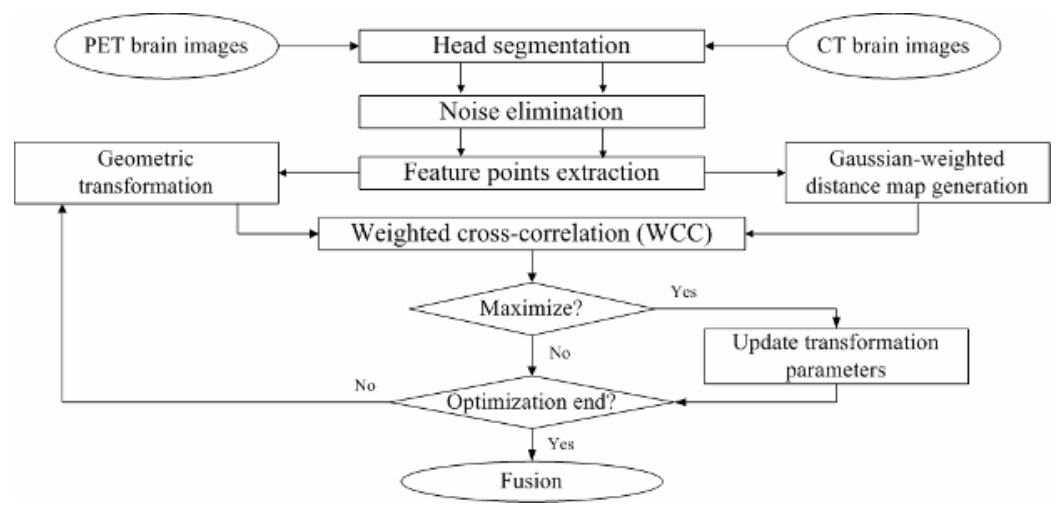

Fig. 1. The pipeline of proposed method using a Gaussian-weighted distance map

First, our 3D IRG starts by choosing a seed voxel at $(0,0,0)$ on whole volume and compares seed voxels with neighboring voxels. Region is grown from the seed voxel by adding neighboring voxels that are less than chosen tolerance. When the growth of region stops, this region is background except head. Then we simply segment the head by inverse operation. Thus our 3D IRG segments the head automatically without holes and the distortion of edges by morphological operations in PET and CT images. Fig. 2 shows the comparison of threshold-based method and our 3D IRG method in PET and CT brain images. In Fig. 2(a) and (c), we can easily see holes inside of the head, whereas our method can clearly segment the head without holes as shown in Fig. 2(b) and (d).

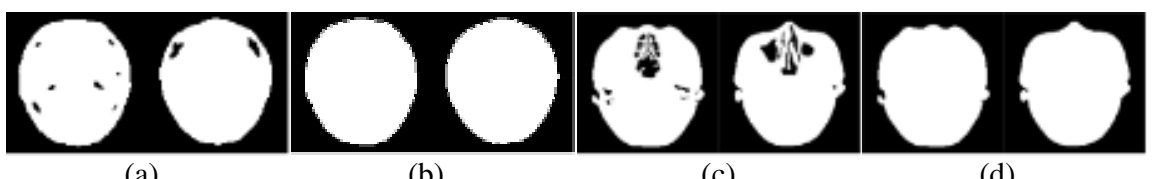

(a) (b)

(c)

(d)

Fig. 2. The comparison of head segmentation between threshold-based method and 3D IRG method in PET and CT brain images (a) and (c) shows the results of the threshold-based method in PET and CT brain images, respectively. (b) and (d) shows the results of our 3D IRG method in PET and CT brain images, respectively.

\subsection{Non-head Elimination Using Region Growing-Based Labeling}

Although the 3D IRG segments the head without holes, the non-head regions having the intensities which are similar to the head can be segmented on background area. Since the size of these non-head regions is small in comparison with the head, we propose a region growing-based labeling (RGL) to efficiently eliminate the non- head regions by removing other regions except the largest region.

Our RGL finds the position of 1's voxel for choosing the seed on the binary images while scanning from position at $(0,0,0)$ to whole volume size. The region is then grown from the seed voxel by adding neighboring voxels based on connectivity and the voxels of growing region are given to label. When the growth of region stops, we identify the size of label. Since the RGL doesn't require any equivalence table and 
renumbering of label, Our RGL provides efficient labeling in comparison with a conventional connected component labeling [7] in memory use and time complexity. As shown in Fig. 3(a) and (c), non- head regions are included in PET and CT brain images. Fig. 3(b) and (d) shows the results of the head without the non-head regions removed by the RGL in PET and CT brain images, respectively.

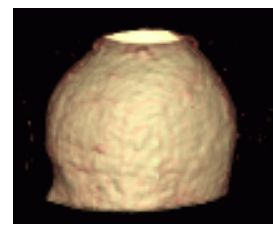

(a)

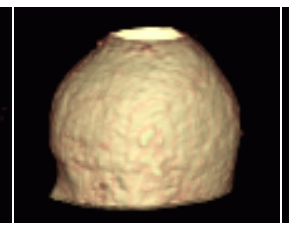

(b)

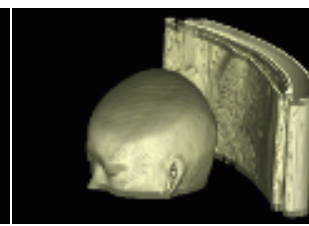

(c)

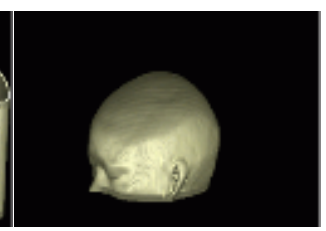

(d)

Fig. 3. The results of the non-head elimination using our RGL method (a) PET brain images (b) the results of non-head elimination in the PET brain image (c) CT brain images (d) the result of non-head elimination in the CT brain image

The feature points are extracted from the binary segmentation images by applying a conventional sharpening filter [7]. Since the holes within head area or the non-head regions in background area are filled or eliminated by 3D IRG and RGL, the feature points are selected from the only head boundary. Fig. 4 shows the feature points of head extracted from PET and CT images, respectively.

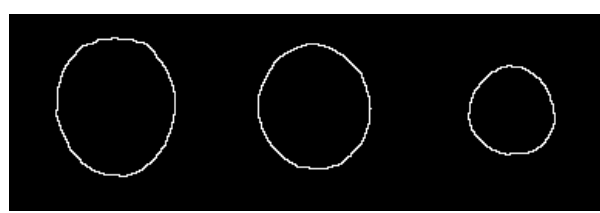

(a)

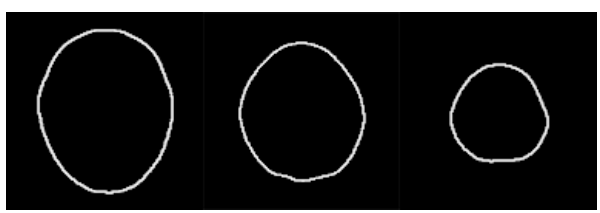

(b)

Fig. 4. The feature points of head extracted from PET and CT images (a) PET slice (b) CT slice

\subsection{Feature Points Extraction and Gaussian-Weighted Distance Map Generation}

A conventional surface registration is likely to lead the similarity measure to converge on the local optimum near to global optimum since the correspondence of the feature points extracted from PET images can differ from the feature points of CT images. To prevent this occurrence we propose the 2D Gaussian-weighted distance map (GWDM) to robustly converge on global optimum even in blurry and noisy images as well as in a large geometrical displacement.

Our 2D GWDM is generated by assigning the Gaussian-weighted mask to the corresponding feature points. If the current weighting is larger than the weighting of neighbor feature points, the previous weighting is changed to the current one. In our method, GWDM is generated only for CT images. The Gaussian-weighted mask is defined as Eq. (1).

$$
G(x, y)=\frac{1}{2 \pi \sigma^{2}} e^{-\frac{1}{2 \sigma^{2}}\left\{\left(x-c_{x}\right)^{2}+\left(y-c_{y}\right)^{2}\right\}} \approx \lambda e^{-\frac{1}{2 \sigma^{2}}\left\{\left(x-c_{x}\right)^{2}+\left(y-c_{y}\right)^{2}\right\}}
$$


where $\sigma$ is set in proportion to the mask size as a standard deviation. $\lambda$ is a scaling parameter. $c_{x}$ and $c_{y}$ is the center of the Gaussian-weighted mask. The weighting of mask is very large at center, and is reduced in proportion to the distance far from center depending on Gaussian curve. $G$ is the Gaussian-weighted mask.

Fig. 5 shows the process for the generation of GWDM in CT brain image. Fig. 5(a) and Fig. 5(b) show the Gaussian-weighted curve and mask with 13 by 13 size, $\lambda=1$, $\sigma=3.0$, respectively. Fig. 5(c) shows the extracted feature points. Fig. 5(d) shows the GWDM generated from feature points. Fig. 5(e) shows the weighting of the GWDM in a magnification of Fig. 5(d). The area corresponding to feature points has the brightest intensities while the area far from feature points has dark ones.

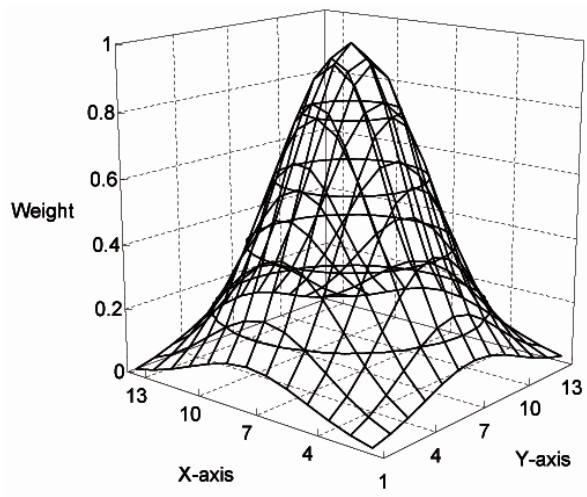

(a)

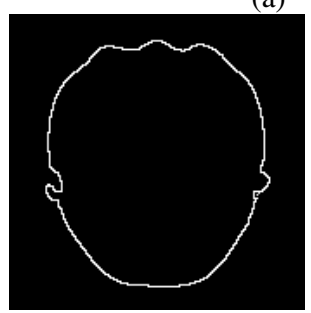

(c)

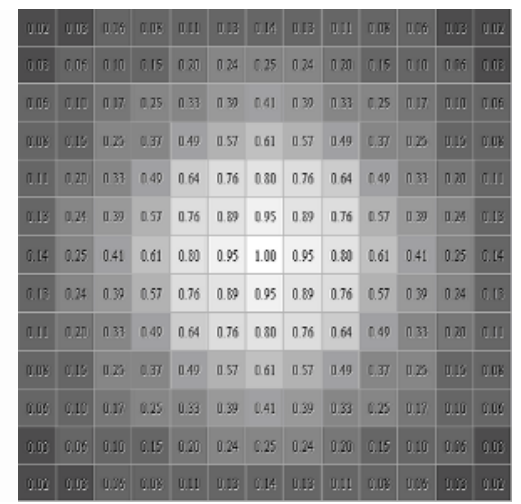

(b)

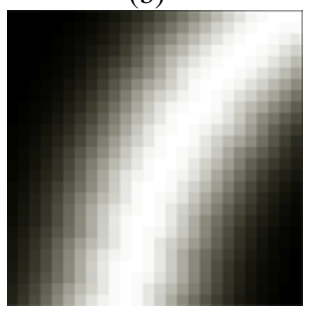

(e)

Fig. 5. The generation of a $2 D$ GWDM in CT brain image (a) the Gaussian curve (b) the Gaussian-weighted mask (c) the feature points of head (d) 2D GWDM (e) magnification of (d)

\subsection{Weighted Cross-Correlation and Optimization}

For similarity measure between the feature points of PET images and the GWDM of CT images, we propose the weighted cross-correlation (WCC). Our approach reduces the computation time because of using the only GWDM of CT images corresponding to the feature points of PET images instead of using whole CT volume. The WCC is defined as Eq. (2).

$$
W C C=\frac{1}{\lambda N_{P E T}} \sum_{i=0}^{N_{P E T}} G_{C T}\left(\operatorname{Tr}\left(P_{P E T}(i)\right)\right)
$$


where $N_{P E T}$ and $P_{P E T}(i)$ are the total number of feature points and the position of $i$-th feature point in PET images, respectively. $T r$ is rigid transformation matrix transforming feature points of PET images into the coordinate system of CT images. $G_{C T}$ is the GWDM of CT images corresponding feature points in PET images. $\lambda$ is a scaling parameter.

In order to search for the optimal location, we find optimal parameters such as $T_{x}$, $T_{y}{ }^{\prime}, T_{z}{ }^{\prime}, R_{x}{ }^{\prime}, R_{y}{ }^{\prime}, R_{z}$ ' when the WCC reaches maximum as following Eq. (3). Powell's multidimensional direction method is then used to maximize WCC. This method searches for optimal location in the order following $T_{x}, T_{y}, R_{z}, R_{x}, R_{y}, T_{z}$ until WCC doesn't change any more and iterate over constant number.

$$
\left(T_{x}{ }^{\prime}, T_{y}{ }^{\prime}, T_{z}{ }^{\prime}, R_{x}{ }^{\prime}, R_{y}{ }^{\prime}, R_{z}{ }^{\prime}\right)=\arg \max (W C C)
$$

\section{Experimental Results}

All our implementation and test were performed on an Intel Pentium IV PC containing 3.2 GHz CPU and 2.0 GBytes of main memory. Our method has been successfully applied to five clinical datasets and two software phantom datasets, as described in Table 1, for evaluating with the aspects of visual inspection, accuracy, robustness, and computation time.

Table 1. Experimental datasets

\begin{tabular}{ccccccc}
\hline \multirow{2}{*}{ Dataset } & CT/PET & $\begin{array}{c}\text { Image } \\
\text { size }\end{array}$ & $\begin{array}{c}\text { Slice } \\
\text { number }\end{array}$ & $\begin{array}{c}\text { Voxel Size } \\
(\mathrm{mm})\end{array}$ & $\begin{array}{c}\text { Slice spacing } \\
(\mathrm{mm})\end{array}$ & $\begin{array}{c}\text { Intensity } \\
\text { range }\end{array}$ \\
\hline \hline \multirow{2}{*}{ Patient1 } & CT & $512 \times 512$ & 158 & $0.38 \times 0.38$ & 1.0 & $0 \sim 4095$ \\
& FDG-PET & $128 \times 128$ & 40 & $1.95 \times 1.95$ & 3.33 & $0 \sim 255$ \\
\hline \multirow{2}{*}{ Patient2 } & CT & $512 \times 512$ & 35 & $1.17 \times 1.17$ & 5.00 & $-976 \sim 1642$ \\
& FDG-PET & $128 \times 128$ & 82 & $2.00 \times 2.00$ & 2.00 & $0 \sim 4095$ \\
\hline \multirow{2}{*}{ Patient3 } & CT & $512 \times 512$ & 34 & $1.17 \times 1.17$ & 5.00 & $48 \sim 2857$ \\
& FDG-PET & $128 \times 128$ & 45 & $4.00 \times 4.00$ & 4.00 & $0 \sim 4095$ \\
\hline \multirow{2}{*}{ Patient4 } & CT & $512 \times 512$ & 28 & $1.17 \times 1.17$ & 5.00 & $-976 \sim 1933$ \\
& FDG-PET & $128 \times 128$ & 80 & $2.00 \times 2.00$ & 2.00 & $0 \sim 4095$ \\
\hline \multirow{2}{*}{ Patient5 } & CT & $512 \times 512$ & 37 & $1.17 \times 1.17$ & 5.00 & $48 \sim 4048$ \\
& FDG-PET & $128 \times 128$ & 53 & $4.00 \times 4.00$ & 4.00 & $0 \sim 4095$ \\
\hline Software & CT & $128 \times 128$ & 40 & $1.95 \times 1.95$ & 3.33 & $0 \sim 2224$ \\
phantom 1 & FDG-PET & $128 \times 128$ & 40 & $1.95 \times 1.95$ & 3.33 & $0 \sim 4095$ \\
\hline Software & CT & $128 \times 128$ & 40 & $1.95 \times 1.95$ & 3.33 & $498 \sim 2721$ \\
phantom2 & FDG-PET & $128 \times 128$ & 40 & $1.95 \times 1.95$ & 3.33 & $0 \sim 4095$ \\
\hline
\end{tabular}

As shown in Fig. 6, PET software phantom datasets simulate background, tissue, and brain in the head and are generated by using Gaussian smoothing for blurry properties. The standard deviation of Gaussian smoothing in PET software phantom1 and phantom 2 are 1.0 and 2.0, respectively. CT software phantom datasets simulate four areas such as background, tissue, muscle, and skull. In particular, the Gaussian noise with standard deviation 20.0 is added to CT software phantom2. We can see that software phantom 2 shown in Fig. 6(c) and (d) are more blurry and noisy than software phantom1 shown in Fig. 6(a) and (b). 


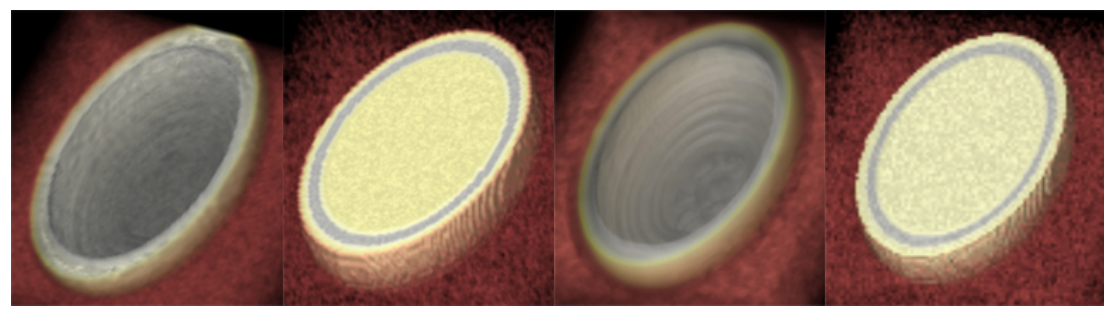

(a) (b)

(c)

(d)

Fig. 6. Software phantom datasets for accuracy and robust evaluation (a) PET software phantom1 (b) CT software phantom1 (c) PET software phantom2 (d) CT software phantom2

Fig. 7 and Fig. 8 show the comparison of 2D visual inspection and 3D fusion before and after registration. In Fig. 7, the results of $2 \mathrm{D}$ visual inspection are displayed by fusing skull edges of CT images and transformed PET brain images in axial, coronal, and sagittal planes together, whereas Fig 8 fuses brain boundary of PET images on the CT images. While the top row of Fig. 7 and Fig. 8 applying scale parameters before registration are misaligned between PET brain images and CT images, the bottom row of Fig. 7 and Fig. 8 applying optimal parameters after registration are well aligned within skull area of CT image. Fig. 7(d) and Fig. 8(d) show the brain in arbitrary $3 \mathrm{D}$ view before and after registration. Fig. 9 shows the aligned results in arbitrary 2D plane and 3D view of clinical datasets after registration.
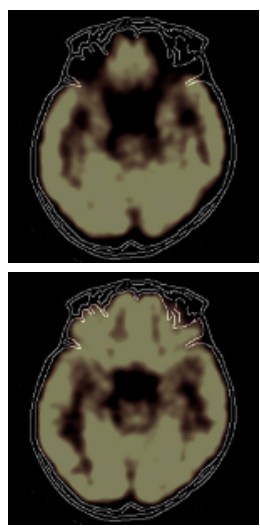

(a)
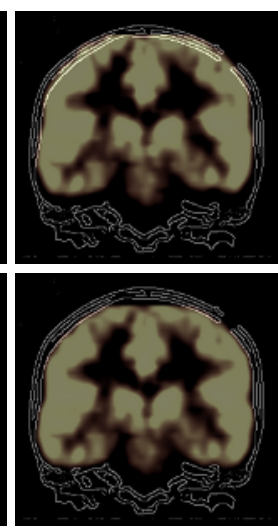

(b)
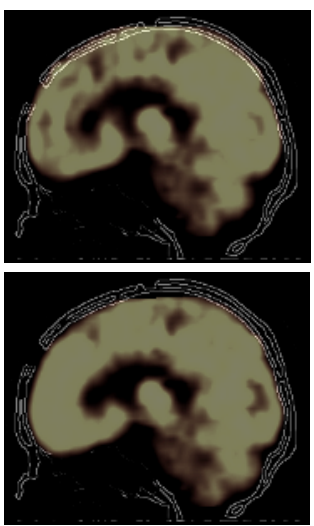

(c)
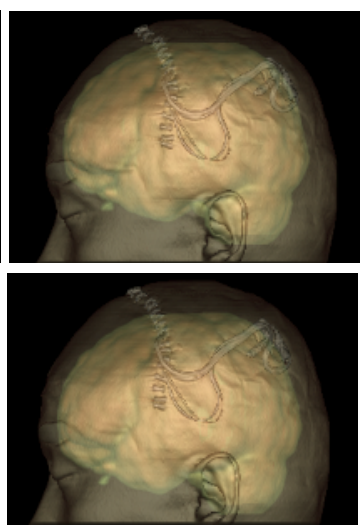

(d)

Fig. 7. The comparison of $2 \mathrm{D}$ visual inspection and $3 \mathrm{D}$ fusion before and after registration in clinical dataset1 (a) axial plane (b) coronal plane (c) sagittal plane (d) 3D fusion

The registration accuracy of our method is evaluated by comparing with the conventional ones such as mutual information (MI)-based registration, chamfer distance matching (CDM), and feature-based cross-correlation (FCC). For the evaluation, we use the software phantom with the known parameters, called as true transformations. In order to quantify the registration error shown in Table 2, we 

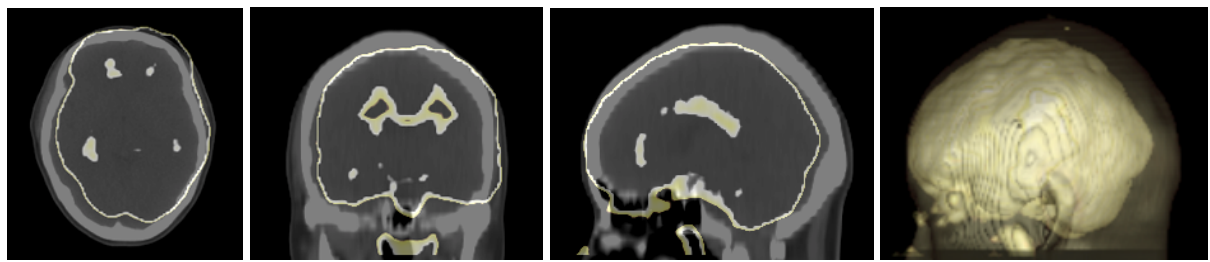

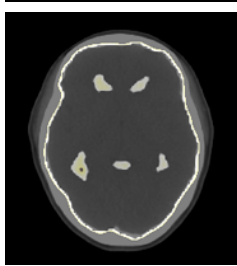

(a)

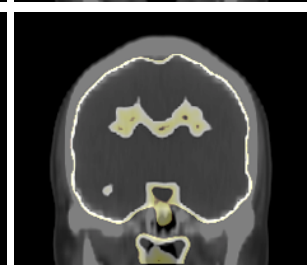

(b)

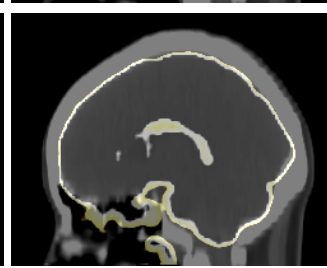

(c)

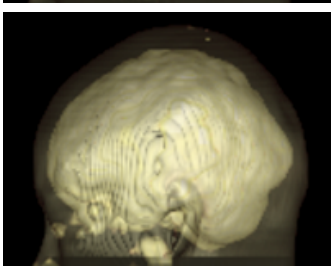

(d)

Fig. 8. The comparison of $2 \mathrm{D}$ visual inspection and $3 \mathrm{D}$ fusion before and after registration in clinical dataset2 (a) axial plane (b) coronal plane (c) sagittal plane (d) 3D fusion
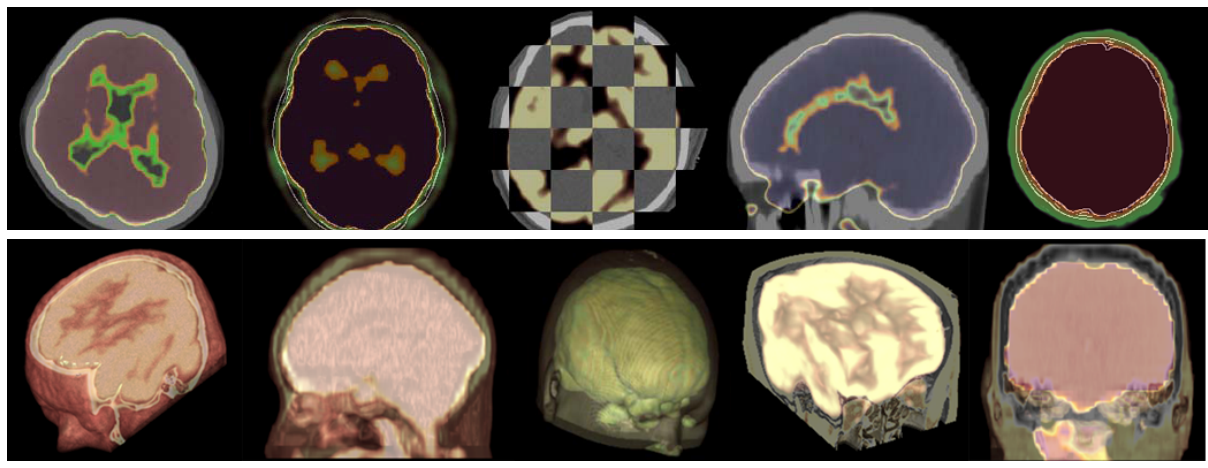

Fig. 9. The results of 2D visual inspection and 3D fusion of clinical datasets after registration

compute each RMSE for translations and rotations as Eq. (4) between estimated parameters and true transformations. At this time, the feature points of head are extracted by applying proposed IRG and RGL for comparing our WCC with CDM or FCC in same environments. The use of MI for accuracy test is restricted to the intensities of whole volume without extracting the feature points of head, and is not included sampling and multi-resolution optimization.

$$
\begin{aligned}
& T-R M S E=\sqrt{\frac{1}{3}\left\{\left(T_{x}-T_{x}{ }^{\prime}\right)^{2}+\left(T_{y}-T_{y}{ }^{\prime}\right)^{2}+\left(T_{z}-T_{z}{ }^{\prime}\right)^{2}\right\}} \\
& R-R M S E=\sqrt{\frac{1}{3}\left\{\left(R_{x}-R_{x}{ }^{\prime}\right)^{2}+\left(R_{y}-R_{y}{ }^{\prime}\right)^{2}+\left(R_{z}-R_{z}{ }^{\prime}\right)^{2}\right\}}
\end{aligned}
$$

In our method, T-RMSE and R-RMSE are less than $0.1 \mathrm{~mm}$ and $0.4^{\circ}$, respectively in two software phantom datasets and give better accuracy than the conventional ones. In particular, MI shows a large different in software phantom2. This means that MI has a limitation in exact alignment when blurry and noisy images are aligned. 
Table 2. Accuracy results using the software phantom with the known parameters

\begin{tabular}{cccccccccc}
\hline Dataset & Method & $\begin{array}{c}\mathrm{T}_{\mathrm{x}} \\
(\mathrm{mm})\end{array}$ & $\begin{array}{c}\mathrm{T}_{\mathrm{y}} \\
(\mathrm{mm})\end{array}$ & $\begin{array}{c}\mathrm{T}_{\mathrm{z}} \\
(\mathrm{mm})\end{array}$ & $\begin{array}{c}\mathrm{R}_{\mathrm{x}} \\
\left({ }^{\circ}\right)\end{array}$ & $\begin{array}{c}\mathrm{R}_{\mathrm{y}} \\
\left({ }^{\circ}\right)\end{array}$ & $\begin{array}{c}\mathrm{R}_{\mathrm{z}} \\
\left({ }^{\circ}\right)\end{array}$ & $\begin{array}{c}\mathrm{T}- \\
\mathrm{RMSE} \\
(\mathrm{mm})\end{array}$ & $\begin{array}{c}\mathrm{R}- \\
\mathrm{RMSE} \\
\left({ }^{\circ}\right)\end{array}$ \\
\hline \hline \multirow{3}{*}{ Software } \\
Phantom & TRUE & 10.73 & 12.48 & -10.14 & -4.8 & -5.2 & 7.3 & - & - \\
\cline { 2 - 10 } 1 & WCC & 10.72 & 12.46 & -10.19 & -4.74 & -5.01 & 7.21 & 0.03 & 0.12 \\
& CDI & 10.71 & 12.64 & -10.34 & -5.31 & -5.55 & 7.67 & 0.14 & 0.42 \\
& FCC & 7.87 & 12.07 & -9.75 & -4.38 & -6.13 & 4.50 & 0.53 & 1.72 \\
\hline \multirow{3}{*}{ Software } & TRUE & -6.83 & -8.58 & 6.24 & 4.8 & -3.2 & -6.3 & - & - \\
Phantom & WCC & -6.75 & -8.59 & 6.39 & 4.43 & -2.69 & -6.19 & 0.10 & 0.37 \\
2 & MI & -7.80 & -8.85 & 5.85 & 5.62 & -5.46 & -7.12 & 0.63 & 1.47 \\
& CDM & -6.22 & -8.53 & 6.70 & 4.19 & 1.00 & -4.88 & 0.44 & 2.58 \\
& FCC & -13.07 & -2.33 & 0.76 & 2.66 & -11.98 & 0.07 & 5.99 & 6.38 \\
\hline
\end{tabular}

For robustness test, we evaluated whether the WCC similarity measure searches for optimal location against the noise in software phantom1 with a large geometrical displacement. White zero-mean Gaussian noise with standard deviation 0, 100, 300, and 500 is superimposed onto the only CT software phantom1. As shown in Fig. 10, increasing the noise level does not affect the maximal WCC at optimal location $(0 \mathrm{~mm}$ or $0^{\circ}$ ), as the position of maximal WCC in traces computed for all six optimal parameters is not changed when the amount of noise is increased. This means that our WCC leads to a global maximum using the GWDM even though feature points extracts differently between PET and CT brain images due to blurry or noisy properties.

The total computation time including 3D fusion in two software phantom datasets is measured by comparing our method with conventional ones in Table 3. Our method gives similar computation time to the CDM and FCC and much faster than the MIbased registration.

Table 3. Total computation time

\begin{tabular}{ccccc} 
& & & & $(\mathrm{sec})$ \\
\hline & WCC & MI & CDM & FCC \\
\hline \hline Software-phantom1 & 8.234 & 391.843 & 8.579 & 8.062 \\
Software-phantom2 & 8.406 & 407.734 & 8.687 & 7.890
\end{tabular}

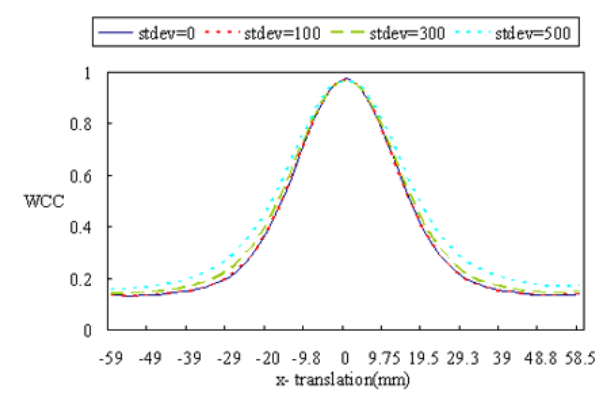

(a)

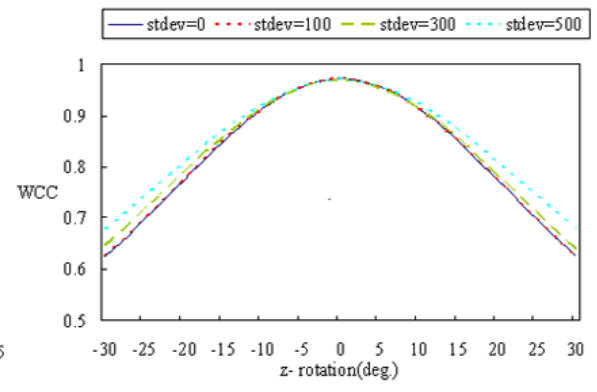

(b)

Fig. 10. The robustness test of WCC in software phantom 1 added the Gaussian noise with standard deviation $0,100,300,500$ (a) translation of $x$-direction in the range from -60 to $60 \mathrm{~mm}$ (b) rotation around z-axis in the range from -30 to $30^{\circ}$ 


\section{Conclusion}

We have developed an accurate and robust surface registration method using a Gaussian-weighted distance map for brain PET-CT fusion. Our 3D IRG segmented the head without any additional processing such as hole filling. The proposed RGL eliminated efficiently the non-head regions in comparison with the conventional connected component-based labeling. Our GWDM led our similarity measure to robust convergence on the optimal location even though feature points extract differently between PET and CT brain images due to blurry or noisy properties. The WCC rapidly measure the similarities because of considering the GWDM of CT images corresponding to the feature points extracted from PET images instead of using whole volume of CT images. Experimental results showed that our method was much faster than MI and more accurate than conventional registration methods such as MI, CDM, and FCC. In particular, our method was robustly registered at optimal location regardless of increasing noise level.

\section{Acknowledgements}

This work was supported in part by a grant B020211 from Strategic National R\&D Program of Ministry of Science and Technology and a grant 10014138 from the Advanced Technology Center Program. The ICT at Seoul National University provides research facilities for this study.

\section{References}

1. J.B.A.Maintz, M.A.Viergever, A survey of medical image registration, Medical Image Analysis, Vol.2, Iss.1 (1998) 1-36.

2. J.Hongjian, R.Richard A., H.T.Kerrie S., New approach to 3-D registration of multimodality medical images by surface matching, Proc. SPIE, Vol.1808, 196-213

3. J.B.A.Maintz, P.A. van den Elsen, M.A.Viergever, Comparison of edge-based and ridgebased registration of CT and MR brain images, Medical Image Analysis, Vol.1, Iss.2 (1996) 151-161

4. F.Maes, A.Collignon, G.Marchal, P.Suetens, Multimodality Image Registration by maximization of Mutual Information, IEEE Transaction on Medical Imaging, Vol.16, No.2 (1997) 187-198.

5. L.Y.Hsu, M.H.Loew, Fully automatic 3D feature-based registration of multi-modality medical images, Image and Vision Computing Vol.19 (2001) 75-85.

6. E.A.Firle, S.Wesarg, C.Dold, Fast CT/PET registration based on partial volume matching, International Congress Series Vol.1268 (2004) 1440-1445

7. R.G.Gonzalez, R.E.Woods, Digital Image Processing, 1st Ed. (1993) 